\title{
Características epidemiológicas de las infecciones invasoras por Haemophilus influenzae tipo b, en el área norte de Santiago
}

\author{
Alfredo Avendaño B. 1; Rosanna Lagos Z.2; Santiago Topelberg V.2; \\ Patricio Herrera L. '; Jean Pierre Dattas L. 2; Pamela Barraza C. 2;

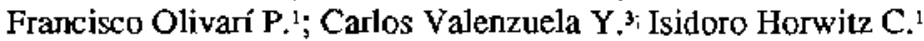 \\ Risk for haousehold contacts of patients with
Haemophilus influenzae type b infections
}

\begin{abstract}
In order to study factors associated with the occurrence of Haemophiflus inthenzoe tipe b i Hibi invasive infections, and to assess the risk of secondary cases in the northern melropolitan area of Santiago, Chile, a controlled study was conducted on 100 households of patients admiled to the locol children's hospilal with Hib diseases and on 100 control households of the same community bearing a child that matched the corresponding case with sespect to age, gender and geographic origin. The families were conseculively enrclled behween april 1989 and december 1991 ; all 200 households were rollowed olong a period of 12 monh, and arlongements wcre made to provide immediale medical care to childien wilh tebrile episodes. Mean attack rale of Hib invasive disease was $19.9 \times 105$ subjects under age 5 years, withoul differences among the populations served by the fourleen ambulatory care focilities of the involved areo. No significanit differences were delected among the study and control household groups regarding to age, gender, socioeconomical level and attendance to school ar day case centers, but both groups differed from the population of the area regarding the propartion on adult members, and the mean number of household members was signiticantly higher in the sludy group than in conlrals 16.9 vs 5.5]. Both Hib colonizotion rate $[7,4$ vs $2.2 \%)$, and the proportion of households wilh al least one col onized member, 130 vs $9 \%$, were higher among household of patients than in controls $(p<0,0001$, and $<0,028$, respectively\}. None of the palienis had a previous conlact with a case of Hib disease, nor secondary cases were cobserved anong their 117 potentially susceplible contocts. On the bosis of a conservative risk, estimole 500 times higher than the locol attack rale, the risk of secondary Hib disease in that area of the city is significanlly different from thal reported in the literalure $(p<$ 0,00081 .
\end{abstract}

(Koy wordsi Hemophilus influenzoe, household contacls, secondary cases, conlact tracing, communicable disease, conlrol, prevention.)

Haemophilus influenzae tipo b (Hib) es uno de los principales agentes eliológicos involucrados en infecciones graves de la infancia, y la primera causade meningitis no epidémica entre los menores de 5 años ${ }^{1-3}$. En paises industrializados, las enfermedades causadas por Haemophilus influenzae

1. Departamento de Pediatría, División Ciencias Médicas Norte, Facultad de Medicina, Universidad de Chile.

2. Servicio de Pediatría. Hospital Roberto del Río, Servicio de Salud Metropolitano Nonte.

3. Departamento de Biología Celular y Genética, Facultad de Medicina, Universidad de Chile.

Estudio financiado por proyecto FONDECYT 1025-89. tipo b son reconocidas como un problema relevante en salud pública y han motivado importantes esfuerzos destinados a conocer sus caracteristicas epidemiologicas y desartollar estrategias para su prevención. Inicialmente éstas últimas se concentraron en torno a la quimioprofilaxis de casos secundarios, descritos especialmente en comunidades norteamericanas y curopcas ${ }^{46}$. Más recientemente los recursos se han canalizado al perfeccionamiento y la evaluación de vacunas para evitar casos primarios ${ }^{3,7-9}$.

En Chile, la importancia clínica de las infeccioncs invasoras por $\mathrm{Hib}$ ha sido ampliamente documentada ${ }^{10-12}$. Sin embargo, el conocimiento del comportamiento poblacional de estas en- 
fermedades es escaso y la información se limita a un estudio retrospectivo en cl área metropolitana".

Puesto que el comportamicnto de las enfermedades infecciosas puede ser muy variable de un país a otro y la planificación de intervenciones de trascendencia comunitaria exige un conocimiento acabado de la epidemiología local, en el Hospital Roberto del Río de Santiago se réalizó un estudio prospectivo, destinado a conocer los aspectos epidemiológicos generales de las enfermedades invasoras por Hib en cl área Norte de Santiago, analizar factores asociados a la ocurrencia de la enfennedad primaria y evaluar cl riesgo de infección secundaria en los contactos de los pacientes en dicha comunidad.

\section{Pacientes y Método}

El trabajo abarcó el periodo comprendido entre abril de 1987 y dicienbre de 1991, c incluy $\delta$ los grupos familia tes de 100 pacientes consccutivamente ingresados con enfermedad grave por Hib bacteriológicamente documentada y de $\mathbf{l} 00$ controlcs sanos, parcados con los antcriones por edad, sexoy consultorio de origen. El discño y la melodología de esıa investigación han sido cxpuestes en detalle en una publica. ción previa ${ }^{13}$.

Origen de tos casos/grupos controles. Los 100 casos cortesponden a pacientes con documentación bacteriológica de enfermctad invasora por Ilib, consecutivamente atcondidos en cl Hospital Ruberto del Rio del Servicio de Salud Mctropolitano Norte. (SSMO), cnire abril de 1987 y diciembre de 1991 . Durante la semana siguiente a cada hospitalización se concurnó al consultorio de origen del paciente y, luego de comprobar su domicilio, se extrajcron del tarjetero los datos de un niño de la misma cdad y sexo, domiciliado al menos a 10 cuadras de distancia y sin parcntesco, ni otro tipo de relación, con el enfermo. A continuación se visitó ambos hogares -eldel caso índice (GFI) y el del caso control (GFC) y sc aplicó una encuesta que incluyó número y edad de los habitantes de la casa; tipo de barrio y construcción de la vivicnda; ocupación, ingresos y escolaridad del jefe de familia. De acuerdo a estas varíables, las familias fueron clasificadas según el método de Graffar modificado ${ }^{14}$. En los primeros 58 casos se visitó además una de las familias que habjtaban la cosa vecina inmediata a la del paciente (GFV). En todos Jos hogares encucstados se practicaron cultivos faringcos a todos los habitantes de la vivienda. La variable "asisiencia a salas cunas o jatdines infantiles" se invesligö encucstando a los casos índjce y a un múmero equivalente al doble de riños controles de la comunidad. Cuandoel paciente asistia a algún tipo de centro de cuidado diumo, se visitó al establecimiento correspondicnte y averiguó la ocumencia de cualquier cniermedad grave, entre los niños matriculados, durante los tres mcses previos y hasta 6 meses después de la hospitasización del paciente

Seguimiento. Todos los grupos familiares encucstados fucron visilados cadid dos meses para indagar sobre cpisolios mórbidos y hospializaciones erure los menores de 6 años. Almismo tiempo, los investigadores estimularon y otorgaron facilidadcs para satisfacer la demanda espontánea de consulas por enfemedades licbriles, por un periodo de un año a contar de la hospritalización del caso índice. Además se realizó un seguinicnto bacteriológico de muestras faríngeas de todos los individuos y se investigó mascadores genćlicos eritrocitatios en los pacientes y sus familiares directos (estos resuliados serán comunicados en otras publicaciones).

Estudios microbiológicos. Las muestras faringeas fucron obtenidas por hisopado con tórulas de alginato, transportadas al laboratorio en medio de Siuan y sembradas en agar chocolate, con $5 \%$ de sangre de caballo, bacitracina (300 $\mu g-m l)$ y $1 \%$ de IsovitalezQ. Laidentificación de Haemophitus influenzae se realizó de acuerdo a los procedimentos habituales y la presencia de cápsula fue determinada mediante coaglutinación con Staphilococcus aweis (cepa Cowan $\Pi$ ), sensibilizado con antisueros Difco polivalentes y monovalentes $^{15}$. Los estudios de susceptibilidad a antibióticos se basaron en el método de Kirby Baver. En todas las cepas invasoras se investigó producción de betalactamasa mediante ténica de cefalosporina cromógena y de cloramfenicol acetil transferasa, por bioenesayo con eepa de $E$. coli ATCC $25922^{15}$

Anślisis de los datos. Como estadísticas descriptivas sc utilizaron porcentajes, promedios y tasas (referidas al núncto de casos por $10^{5}$ habitantes menores de 5 años). Las estadísticas de dispersión fueron expresadas como crror estándar e intervalos de conflanza de $95 \%$. Para las pruebas de signifjcación se usaron los métodos de $t$ de Student pa ta muestras pareadas, chi cuadrado o Fishes de probabilidad exacta y de tistribución binomial. El nivel de significación clegido fue $\mathrm{p}=<\mathbf{0 , 0 5}$ unilateral. El análisis estadístico fue realizado mediante un sistema logico $\mathrm{HMDP}$.

\section{Resultados}

Las formas de presentación clínica de $\operatorname{los} 100$ casos y los respectivos sitios de aislamiento del patógeno se describen en la tabla 1; la distribución según sexo y edad de los pacientes en la tabla 2. Veinticinco de las 100 cepas aisladas eran productoras de betalactamasas y resistentes a ampicilina; cuatro de ellas resultaron, además, productoras de cloramfenicol acetil transferasa y resislentes a cloramfenicol.

El estudio abarcó un período de 50 meses: mayo de 1987 a dicicmbre de 1991 . Veintisiete de los 100 pacientes provenían de áreas gcográficas ajenas al SSMN; excluidos estos casos, la tasa promedio anual de enfermedad invasora por Hib cn cl área norte de Santiago fue de 19,9 por $10^{5}$ menores de cinco años (IC 95\%: 19,95 a 19,97), y las tasas anuales fucron de 18,$1 ; 19,4 ; 22,4$ y 19,8 para los anos $88,89,90$ y 91 , respectivamente. La incidencia de enfermedad invasora fue similar en 
Tabla 1

Formas de presentación clínica y sitio de aislamiento en 100 casos de infeccion invasora por Haemophilus influenzae tipo b

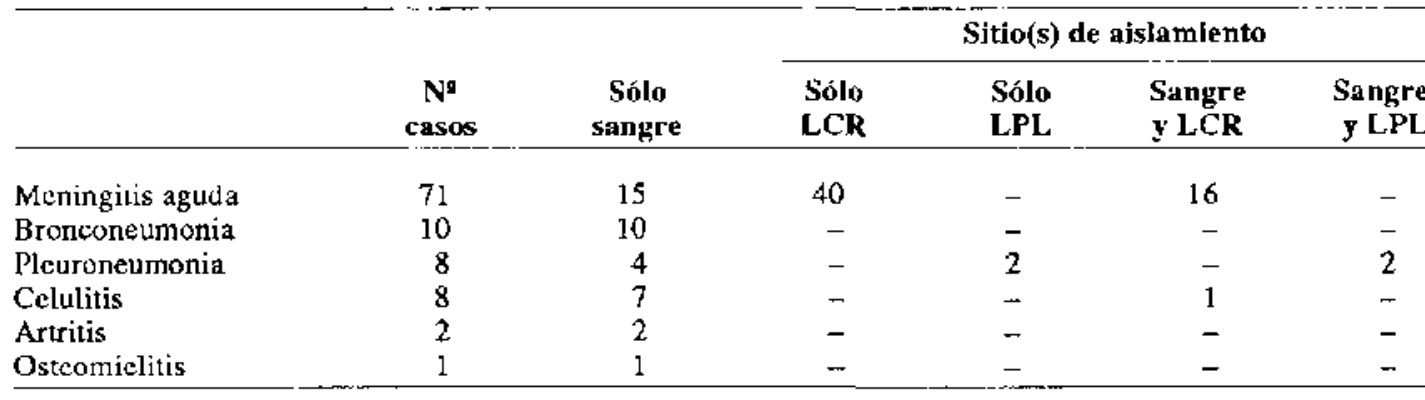

LCR: Líquido cefalo raquídeo. LPL: Líquido pleural.

Tabla 2

Distribución etaria y por sexo en 100 casos de infección invasora por Haemophilus influenzae ripo b

\begin{tabular}{lccc}
\hline $\begin{array}{l}\text { Edades } \\
\text { (años) }\end{array}$ & Varones & Mujeres & \%o de casos \\
\hline $0,001-0,06$ & 11 & 11 & 22 \\
$0,061-1,00$ & 23 & 19 & 42 \\
$1,001-1,06$ & 7 & 9 & 16 \\
$1,061-2,00$ & 2 & 8 & 10 \\
$2,001-3,00$ & 2 & 1 & 3 \\
$3,001-5,00$ & 3 & 1 & 4 \\
$5,0010>$ & 2 & 1 & 3 \\
\hline
\end{tabular}

las zonas geográficas correspondientes a los 14 consultorios del área Norte, excepto en cl Consultorio Colina, donde se observó un incremento significativo de la tasa promedio para el periodo $\left(37,0\right.$ por $10^{5}$, IC $95 \%: 36,7$ a 37,7$)$; esta diferencia ocurrió a expensas de un incremento del número de casos en los años 1988 y $1990(56,9$ y 62,18 por $10^{5}$ menores de 5 años, respectivamentc). Pese a que durante todo el estudio se observó una acumulación, cercana a $50 \%$ de los casos del ano, en el período comprendido entre abril y julio, csta tendencia no alcanzó significación estadisti$c a(p>0,12)$.

Lacomposición etariay por sexos de las familias estudiadas se expone en la tabla 3. De acuerdo a lo esperado, la distribución por edades de los integrantes de los GFI y GFC fue similar; en ambos casos difirio de la población general del SSMN ${ }^{16}$, a consecuencia de mayor proporción de adultos en esta última $y$ de individuos menores de 6 años en las dos primeras. Los sujetos en edad escolar (de 6 a 10 años) cstuvieton igualmente representados en la población general del área Norte y en las dos muestras estudiadas; del mismo modo la proporción de familias con uno o más integrantes en esta categoria etaria fuc similar en los grupos índice y controles. La distribución por edades de las familias vecinas fue similar a la descrita para la población del SSMN'16. Por otra parte, las proporciones de casos y controles que asistían a salas cunas y jardines infantiles fucron similares ( 8 y $9 \%$ respectivamente; $p>0,4)$.

El promedio de habitantes y la proporción de familias con más de cuatro integrantes fueron significativamente mayores entre los pacientes que entre los controles $(6,9$ y $5,5 \mathrm{p}<0,0001$, IC $95 \%=0,7$ a 2,$14 ;$ y 79 v $59 \%, p=0,004)$, en tanto que el indicador de Graffar revelo una condición socioeconómica similar en los dos tipos de grupos familiares: 70,9 de los GFI y $66 \%$ de los GFC se ubicaron en las categorías IV y V de este indicador ( $p>0,5$ ), distribución que tampoco difirió de la que ha sido publicada para la población general del SSMN17. El número de personas por dormitorio fue similar en ambos tipos de familias $(x=2,9$ y 2,5 respectivamente).

Las tasas de colonización faringea por Hib fueron significalivamente distintas entre los integráutes de los GFI y los GFC, como también entre los primeros y las 58 familias vecinas (tabla 4); 
Tabla 3

Composición de 100 fanilias "índice", 100 controles y 58 "vecinos", según sexo y edad

\begin{tabular}{|c|c|c|c|c|c|c|c|c|c|}
\hline Edad (años) & $\mathbf{V}$ & $\begin{array}{c}\text { Indices } \\
\text { n: } 691^{\star} \\
\quad M\end{array}$ & $\%$ & $\mathbf{V}$ & $\begin{array}{r}\text { Contro } \\
\text { n: } \mathbf{5 5} \\
\text { MI }\end{array}$ & $\%$ & $\mathbf{v}$ & $\begin{array}{l}\text { Vecinos } \\
\text { n: } \mathbf{3 3 1} \mathbf{1}^{\mathbf{c}} \\
\mathbf{M}\end{array}$ & $\%$ \\
\hline $00,0-02,0$ & 78 & 66 & 20,8 & 57 & 60 & $2 l, 2$ & 18 & 10 & 8,5 \\
\hline $02,1-05,0$ & 38 & 34 & 10,4 & 29 & 27 & 10,2 & 19 & 15 & 10,3 \\
\hline $05,1-10,0$ & 38 & 32 & 10,1 & 28 & 34 & 11,2 & 29 & 20 & 14.8 \\
\hline $10,1-15,0$ & 19 & 18 & 5,4 & 27 & 14 & 7.4 & 6 & 20 & 7,8 \\
\hline$>15,0$ & 165 & 203 & 53,3 & 115 & 160 & 50,0 & 83 & 111 & 58,6 \\
\hline Total & 338 & 353 & & 256 & 295 & & 155 & 176 & \\
\hline
\end{tabular}

a: incluye casos índicc. b: incluye casos control. c: incluye 58 familias.

V: varones. M: mujeres.

Tabla 4

Tasas de portación de Haemophilus influenzae tipo b según lipo de grupo familiar y edad

\begin{tabular}{|c|c|c|c|c|c|c|c|c|c|}
\hline \multirow[b]{2}{*}{ Edad (años) } & \multicolumn{3}{|c|}{ Indices } & \multicolumn{3}{|c|}{ Contrules } & \multicolumn{3}{|c|}{ Vecinos } \\
\hline & $\mathrm{N}^{\mathbf{g}}$ & Port & $\%$ & $N^{\mathbf{9}}$ & Port & $\%$ & $\mathbf{N}^{\mathbf{9}}$ & Port & $\%$ \\
\hline $00,0-02,0$ & 52 & 6 & 11.5 & 33 & 0 & 0,0 & 28 & 0 & 0 \\
\hline $02,1-05,0$ & 65 & 10 & 15,4 & 42 & 2 & 4,8 & 34 & 0 & 0 \\
\hline $05,1-10,0$ & 69 & 10 & 14,5 & 60 & 1 & 1,2 & 49 & 1 & 2 \\
\hline $10,1-15,0$ & 37 & 4 & 10,8 & 41 & 1 & 2.4 & 26 & 3 & 11,5 \\
\hline$<15,0$ & 368 & 14 & 3,8 & 275 & 6 & 2,2 & 194 & 0 & 2,6 \\
\hline Total & $59 l^{\circ}$ & & $7,4^{\mathrm{d}}$ & $451^{b}$ & & & $2,2^{\circ}$ & $331=$ & 1, \\
\hline
\end{tabular}

a : no incluye casos indice

b : no incluye casos control

c : incluye 58 familias

asociada a esta diferencia, en los GFI había mayor proporción de hogares con al menos un miembro colonizado por $\mathrm{Hib}(30$ vs $9 \%, \mathrm{p}<0,028)$. La variable "tiempo de permanencia en el hogar" tuvo un comportamiento similar entre los portadores identificados en los GFI y GFC; en ambos casos, la mayoría ( 87,5 y $91 \%$, respectivamente) de los individuos colonizados refirió haber permanecido en el hogar más de 16 horas diarias, durante la semana previa a la hospitalización del paciente. No obstante, en 16 de los 30 hogares índice se encontró que al menos un portador de Hib compartía el dormitorio con el paciente, situación que se observó en sólo uno de los nueve hogares control $(\mathrm{p}=0,028)$.
En ninguno de los 100 pacientes se encontró antecedente anamnésico de contacto con un caso de enfermedad invasora por Hib. Ninguno de los 116 niños menores de 6 años expuestos a los casos índices en los respectivos hogares o en los centros de cuidado diurno $(n=72$ contactos menores de un año), presentó episodios sugerentes de enfermedad invasora por $\mathrm{Hib}$, en el periodo de scguimiento. En relación a lo publicado en la literatura, sobre la base de una tasa de ataque secundario intrafamiliar (conservadora), 500 veces superior a la de la población general local, la diferencia entre el número de casos esperado $(\mathbf{n}=11,6)$ y observado $(\mathbf{n}=0)$, entre estos 116 contactos susceptibles, resultó altamente significativa $(p<0,000005)$. 
A su vez, en guarderías infantiles, el número esperado $(7,2$ casos), difirió significativamente del observado $(n=0, p<00,0008)$.

\section{Comentario}

En el periodo estudiado (1987-1991), las tasas anuales de infecciones invasoras por $\mathrm{Hib}$ en el SSMN oscilaron entre 18,1 y 22,4 por $10^{5}$ menores de 5 años, cifras que se ubican en el límite inferior de los rangos de incidencia reportados en EE.UU. y Europa ${ }^{18,} 19$. Para el trienio 1985-1987, Fentecio y cols. informaron, en esta misma comunidad, una tasa de 52,5 por $10^{5}$ menores de 5 anfos ${ }^{3}$, más cercana a la descrita en poblaciones nortcamericanas y europeas. En esta muestra, $80 \%$ de los casos ocurrieron en niños menores de 18 meses, hallazgo que reproduce lo reportado para el área metropolitana en general. por los autores antes mencionados. No encontramos explicación para el incremento de casos observados en Colina en los ańos 1988 y 1990 , pero esta mayor incidencia no se asoció a mayor prevalencia de portadores faríngeos de Hib. La meningitis aguda fue la forma clínica y predominante ( $71 \%$ ) y, al igualque en otra serie nacional ${ }^{3}$, la epiglotitis estuvo ausente. La frecuencia relativa de los síndromes clínicos observada en esta casuística difiere de lo notificado en EE.UU. y Finlandia, donde a la meningitis aguda corresponden 60 y $46 \%$ de los casos respectivamente y a la epiglotilis hasta $29 \%$ 19-2I. Esta diferencia pudicra estar asociada a las características locales de las edades de ataque, discutidas en el párrafo anterior; en este sentido las mayores incidencias de epiglotitis han sido reportadas en los países nórdicos, donde las infecciones invasoras por Hib se presentan a edades más tardias ${ }^{19}$, 22. Por otra parte, la menor frecuencia de cuadros pleuropulmonares y osteoarticulares observada en esta serie (con respecto a las norteamericanas y europeas), pudiera corresponder a subdiagnóstico de las formas de localización extrameningeas.

En EE.UU. ha quedado establecido que el contacto con un caso de infección invasora por Hib determina, para los individuos susceptibles, un riesgo de enfermedad sistémica significativamente mayor que el de la población no expuestat6. 23. No obstante, que en dicho país ese factor juega un papel importante en comunidades cerradas, como familias y guarderías infantiles, se reconoce que, cn más de $95 \%$ de los casos, las enfermedades graves por Hib se presentan sin anteccdente de contacto con un caso similar ${ }^{24}$. En esta serie, todos los episodios correspondieron a infecciones primarias y ninguno de los niños susceptibles cxpuestos a estos enfermos desamo116 infección grave por Hib. Esta observación permite sostener que el riesgo de enfermedad secundaria, en el área Norte de Santiago, difiere significativamente del de la comunidad norteamericana. Como una explicación a este fenomeno (en el ambiente intrafamiliar) postulamos que nuestra población se caracterizaría por una exposición ambiental precoz a Hib, la cual deteminaría, por una parte, el patrón epidemiológico de acumulación relativamente temprana de los casos y, por otra, el desarrollo también temprano de inmunidad natural. En esta situación teórica, el niño enfermo representaría el único individuo susceptible del hogar, pese a que habiten en él otros miembros en edad de riesgo. Aunque csta hipótesis no explica la ausencia de casos secundarios entre los contactos de salas cunas y jardines infantiles, la asistencia a esos establecimientos resultó bastante infrecuente entre casos y controles de esta serie.

Los grupos familiares de los pacientes con enfermedad sistémica por Hib (y los de los respectivos controles), presentan una estructura etaria diferente de la población general del SSMN; esto es consecuencia lógica del factor involucrado en la selección de las familias - un micmbro enfermo o en cdad de riesgo de infección invasora por $\mathrm{Hib}-$ el cual determinó sobrerrepresentación de familias jóvenes, compuestas por personas cn edad fértil. Sin embargo, nuestras obscrvaciones se alejan de lo esperado en el comportamiento de algunas de las variables clásicamente asociadas a riesgo de cnfermedad primaria. Estudios extranjeros han scîalado que, en las familias de niños con enfermedad invasora por Hib, la frecuencia de miembros de edad comprendida entre los 6 a 10 años es significativamente más alta que en la población general ${ }^{24,25}$, asociación que es atendible atribuir a la mayor prevalencia de colonización faríngea propia de los niffos pertenecientes a esta categoría etaria. En contraste, los individuos en edad escolar de esta muestra se distribuycron de manerá similar en las familias índice, en sus correspondientes controles, y en la población general. Dado que esta muestra posee poder suficiente para detectar razones de disparidad inferiores a 1,5; concluimos 
que, en la comunidad del SSMN, la presencia de escolares en el hogar no constituye un factor asociado a mayor ricsgo de enfermedad primaria. Por otra parte, la asistencia a centros de cuidado diurno lue igualmente infrecuente entre casos y controles, y por cnde tampoco resultó una variable asociada a incremento de riesgo de infección invasora. En cambio, encontramos una diferencia significativa en la densidad de integrantes de los hogares índice y controles, lo que reproducc observaciones en orras comunidades ${ }^{24,25}$; cn el caso del árca Norte de Santiago, esta asociación no parcce derivar de diferencias en el estrato sociocconónico de las familias, a juzgar por la homogeneidad de la distribución del indicador de Graffar y el promedio similar de personas por domitorio cn los GFI y los GFC. Asimismo, esta experiencia se asemeja a lo reportado en publicaciones extranjeras, respecto de la mayor portación faríngca de Hib en las familias de pacientes con enfermedades invasoras 26,27 , fenómeno que afectó tanto al total de individuos como al número de grupos familiares. Otro hecho interesante fue el hallazago de mayor frecuencia de intimidad entre portadores y casos índices, reflejado en la convivencia en dormitorios, que entre los individuos colonizados por Hib y los correspondientes controles, en los hogares respectivos.

En síntesis, la epidemiología local de las enfermedades graves por Hib difiere on varios aspectos de la descrita en países industrializados. Si bien estas obscrvaciones provienen en una comunidad restringida y pudicran no representar al resto del país, este estudio demuestra que las infecciones invasoras por Hib poseen características regionales propias y, en consecucncia, su comportamiento local no puede ser deducido de información recopilada en otras comunidades. En el caso de infecciones invasoras por Hib, como en cualquier enfermedad infecciosa, la planificación de estrategias de control de trascendencia poblacional exige un conocimiento acabado de la epidemiología local.

\section{Resumen}

Para estudiar factores asociados a la ocurrencia de infecciones invasoras por Haemophilus influenzae tipo b (Hib) y evaluar el riesgo de casos secundarios en la población del área Norte de Santiago, se realizó un estudio controlado en los grupos familiares de 100 pacientes con enfermedad grave por $\mathrm{Hib}$, conseculivamente atendidos cn el Hospital Roberto del Río entre abril de 1989 y dicicmbre de 1991 y de 100 controles sanos pareados por edad y sexo, del consultorio de origen del paciente. En todos los grupos familiares se realizó una encuesta socioeconómica y se practicó un seguimiento de un año, durante el cual se dio satisfacción a la demanda de consultas por episodios febriles entre los menores de 5 años. La tasa de ataque promedio (19,9 por $10^{5}$ menores de 5 años) fue similar en las poblaciones de los 14 consultorios del área de salud. Las familias de enfermos y controles fueron similares en nivel socioeconómico, composición por sexo y cstratificación etaria. El número promedio de integrantes de las familias de casos $(n=6,9)$, fue significativamente mayor que en controles $(n=5,5 ; \mathrm{p}<$ 0,0001 ) y en ambas la proporción de adultos fue inferior a la del área de salud. La tasa de portación de Hib $(7,4$ vs $2,2 \%)$ y la proporción de hogares con algún miembro colonizado ( 30 vs $9 \%$ ), fucron significativamente mayores en las familias de casos que de controles ( $p<0,0001$ y $p<0,028$, respectivamente). Ningún paciente tenía antecedentes de contacto previo con un caso similar y no se observaron casos secundarios entre 117 contactos menores de 5 años. Estimando un riesgo (conservador) 500 veces superior al de la población general, el de enfermedad sccundaria en la comunidad del área estudiada difiere en forma significativa del descrito en otros países $(p<$ 0,0008 ).

(Palabras clave: Haemophilus influenzae, contactos intrafamiliares, casos secundarios, seguimiento de contactos, cnfermedades transmisibles, control, prevención.)

\section{Agradecimientos}

A los directores, enfermeras coordinadoras y auxiliares técnicos paramédicos de los consultorios que abarcó el estudio, cuya ayuda facilitó considerablemente esta tarea. A don Enrique Delgado, por su colaboración en el manejo computacional de la información.

\section{Referencias}

1. Turk $D C$ : Clinical Importance of Hacmophilus influenzae-1981. in: Sell SH and Write (cds): Haemophilus influenzac epidemiology, immunology and prevention of disease. Elsevier Biomedical. New York. Amsterdan, Ox ford 1982; 3-9. 
2. Ward J, Cochi S: Haemophilus influenzae vaccines. en: Ploukin S., Mortiner E. Vaccines. Philadelphia. W.B. Saunders Company, 1988: 300-392.

3. Ferreccio $C$, Ortiz E, Astroza L, Rivera C, Clemens $J$. Levine $M$ : A population-based retrospective assesment of the discase burden resulting from invasive Hre. mophilus influenzae in infants and young children in Sanliago, Chile. Pediatr Infect Dis J 1990; 9: 488-494.

4. Ward J, Fraser D, Baraff $J$, Plikayt is $B$ : Haemophilus influenzac meningitis: a national study of secondary spread in hous ehold contacts. N Engl J Med 1979; 301 : 122-125.

5. Tejane A, Dobias $B$, Nangia $B$, Welkuru $H$ : Intrafamily spread of Haemophilus influenzae type $b$ infections. Am J Dis Child 1977; 131: 778-790.

6. Redmond $S$, Pichichero $M$ : Haemophilus influenzae type b disease: An Epidemiologic Study With Special Reference to Day care Centers. JAMA 1984; $252: 2581$ 2584

7. Ward J, Cochi S: Haemophilus influenzae vaccines. In PloukinS.A., Mortimer A. (eds). Vaccines. Philadelphia: Saunders, 1988: 309-320.

8. Eskola J, Kayhty H. Takala A et al.: A randomized, prospective field trial of a conjugate vaccine in the protection of infants and young children againstinvasive Haemophilus influenzac type b discase. N Engl J Med 1990; 323: 1381-1387.

9. Ferreccio $C$, Clemens $J$, Averdanio A et al.: The clinical and immunologic response of chilean infants to Haemophijus influenzae type b polysaccharide telanus protein conjugate vaccire coadministered in the same syringe with diphteria -tetanus toxoids-pertussis vaccine at two, four and six months of age. Pediatr Infect Dis J 1991; 10: 764-771.

10. Herrera P, Prenzel I, Topelberg S: Infecciones graves por Haemophilus influenzae en el niño. 1. Aspectos generales clínicos. Rev Med Chile 1983; 111 : 808-814.

11. Herrera P, Prenzel I, Vildósola $C$ : Empiemas pleurales por Haemophilus influenżae en niôos. Bol Med Hosp Inf México 1979; 36: 665-677.

12. HerreraP, Vildosola C, Samith S: Neumopatias agudas por Haemophilus influenzae: aspecios clínicos y radiologicos en 210 casos. Rev Chil Pediatr $1985 ; 28$; $7-9$.

13. Avendario A, Horwirz I, Dafias JP et al.: Estudio prospectivo en niños con enfermedades invasivas por Haemophilus influenzae y contactos familiares: casos secundarios y cultivos faríngeos. Diseño de la investigación. Pediatría (Santiago) 1988; 31: 132-135

14. Vaienzuela C, Herrera P: Fenotipos A, B, O en infecciones invasoras por Haemophilus influenzac tipo b. Publicado en IV Congreso Latinoamericano de In. fectología Pedíatrica y VIII Congreso Chileno de Infectología, 23 al 26 de octubre 1991, Santiago (pág. 12).

15. Lagos $R$. Enriquez $N$, Lagosiena $C$ : Dinámica de la portación faríngea de Hacmophilus influenzae tipo ben familias del Area Norte de Sanliago. Publicado en Libro de Resúmenes del IV Congreso Latinoamericano de Infeclología Pediátrica y VIII Congreso Chileno de Infectologia, 23 al 26 de octubre 1991, Santiago (pág. 32).

16. Kilian M. Haemophilus. Laennete EH, Ballows WJ, Chapomy J: In: Manual of Clinical Microbiology, 4n. ed. American Sociates for Microbiology, Washington D.C. 1985: 387-393.

17. Valenzuela J, Diaz E, Klagges B: Empleo de un nuevo método de clasificación social. Cuad Med Sor(Santiago) 1976; 17: 14-22.

18. Departamenio de Informótica. Unidad de Estadistica: Servicio de Salud Metropolítano. (Comunicación personal, en base a datos del DNE).

19. Avendaño $A$, Vaienzuela $C$ : Estudio longitudinal de crecimiento y desarrollo de 6 a 20 años de edad. Area None de Santiago. Pediatría (Santiago) 1988 31: 6.

20. Munson R, Kabeer H, Lenoir $A$ and Granoff D: Epidemiology and Prospects for Prevention of Diseascs Due to Hacmophilus influenzae in Developing Countries. Rev Infect Dis 1989; 11 (Supp. 3): 588-597.

21. Takala K, EskolaJ, Pellota H, Makela H: Epidemiology of invasive haernophilus influenzae type b disease among children in Finland before vaccination with Haemophilus influenzae type b conjugate vaccine. Pediatr Infect Dis J 1989; 8: 297-302.

22. Granoff $D$, Basden $M$ : Haemophilus influenzae infecioons in Fresno county, Califomia: A Prospective Study of the Effects of Age, Race, and Contact with a Case on Incidence of Disease. J Infect Dis 1980; 14:4046.

24. RadnondS, Pichichero $M$ : Haemophilus influenzaetype b disexse. JAMA 1984; 252: 2581-2584.

25. Murphy $T$, Osterholm $M, P$ ierson $L$ et al.: Prospective surveillance of Haemophilus influenzae type b disease in Dallas Counly, Texas, and in Minnesota. Pediatrics 1987; 79: 173-179.

26. Barion L, GranoffD, Baremtamp $S$ : Nosocomial spread of Haemophilus influenzae type binfections documented by outer membrane proteins subtype analysis. J Pdiatr 1983; 103: 820-824.

27. Istre G, Conner J, Broome C. Hightower A, Hopkins $R S$ : Risk factors for primary invasive Haemophilus influenzae disease: Increased risk from day care alten dance and school-aged household members. J Pediatr 1985: 105: 190-195.

28. Oursted $C$ : Haemophilus influenzae meningitis: A possible ecological factor. Lancet 1950; 1: 161.

29. Cochis, Fleming D, Hightower A et al.: Primary invasive Haemophilus influenzae type b disease: A population basal assessment of risk faclors. J Pedialr 1986; 108 : 887-896.

30. Michaels $R$, Norden $C$ : Pharyngeal colonization with Haemophilus inftuenzae type b: a Iongitudinal study of families with a children with meningitis or epiglotitis due to Haemophilus influenzae type b. I Infect Dis $1977 ; 108: 887-896$. 